\title{
Noisy Kuramoto-Sivashinsky equation for an erosion model
}

\author{
Kent Bækgaard Lauritsen, ${ }^{(1,3)}$ Rodolfo Cuerno, ${ }^{(2,3)}$ and Hernán A. Makse ${ }^{(3)}$ \\ (1) Niels Bohr Institute, Center for Chaos and Turbulence Studies, Blegdamsvej 17, 2100 Copenhagen Ø, Denmark \\ (2) Departamento de Matemáticas, Universidad Carlos III de Madrid, Butarque 15, 28911 Leganés, Spain \\ (3) Center for Polymer Studies and Department of Physics, Boston University, Boston, Massachusetts 02215
}

(February 1, 2008)

\begin{abstract}
We derive the continuum equation for a discrete model for ion sputtering. We follow an approach based on the master equation, and discuss how it can be truncated to a Fokker-Planck equation and mapped to a discrete Langevin equation. By taking the continuum limit, we arrive at the Kuramoto-Sivashinsky equation with a stochastic noise term.
\end{abstract}

PACS Numbers 1995: 64.60.Ht, 68.35.Rh, 05.40.+j, 79.20.Rf

\section{INTRODUCTION}

The field of nonequilibrium interface growth is currently being investigated by a variety of approaches [1] 3 . One direction of research is the formulation of Langevin equations which are studied by means of analytical and numerical methods. Typically, the terms appearing in Langevin equations are derived by the use of symmetry arguments. A different approach consists in the formulation of discrete models which contain the relevant physical mechanisms present in the problem at hand. The models are studied by computer simulations in order to determine which universality class they belong to. One can try to relate the models to continuum descriptions. In many occasions such an identification is indirect since it is difficult to derive the continuum description for a given set of microscopic growth rules.

There exist cases in which it is possible to derive Langevin equations describing various discrete growth models [4]. The approach followed in these cases is based on the master equation which determines the evolution of the joint probability density $P(H, t)$, where $H$ specifies the interface configuration and $t$ denotes time. The master equation reads

$\frac{\partial P(H, t)}{\partial t}=\sum_{H^{\prime}} W\left(H^{\prime}, H\right) P\left(H^{\prime}, t\right)-\sum_{H^{\prime}} W\left(H, H^{\prime}\right) P(H, t)$,

where $W\left(H, H^{\prime}\right)$ denotes the transition rate per unit time from configuration $H$ to $H^{\prime}$, and the sums are over all configurations $H^{\prime}$. One can study the associated Fokker-Planck equation and therefrom derive a continuum Langevin equation for the surface height provided the fluctuations in the system are not too large, and provided the continuum limit can be justified [8]. An important feature of the master equation approach is that it provides a relation between the fluctuations (noise) in the system and the parameters in the model.

In the present paper, we follow the master equation approach in order to derive the continuum equation for a simple pattern forming model recently introduced to study the morphology of surfaces eroded by ion sputtering [9]. The equation which we obtain for the evolu- tion of the height profile $h(x, t)$ is a noisy version of the Kuramoto-Sivashinsky (KS) equation and reads

$$
\frac{\partial h}{\partial t}=v_{0}-|\nu| \nabla^{2} h-\kappa\left(\nabla^{2}\right)^{2} h+\frac{\lambda}{2}(\nabla h)^{2}+\eta(x, t),
$$

where $v_{0}, \nu, \kappa$, and $\lambda$ are constants, and $\eta(x, t)$ is a Gaussian white noise. The KS equation (obtained when $\eta=0)$ [10] is often considered a paradigm of spatiotemporal chaos and appears frequently in studies of pattern formation [11]. An initially flat one-dimensional interface described by the KS equation evolves in time from an almost periodic morphology at early times to a rough surface at late times [12 14 described by the KardarParisi-Zhang (KPZ) equation [15].

The model for ion sputtering in Ref. [] shows an initial periodic morphology and a late-time KPZ scaling regime which are similar to the behavior of the KS equation. However, the KS equation is a deterministic equation whereas the erosion model is inherently stochastic. In 9 the early and late time dynamics of the erosion model were numerically studied with the conclusion that they are the same as those obtained from the noisy KS equation [16]. Here, we confirm this result by showing analytically that the noisy KS equation yields the continuum description of the erosion model. By using the master equation approach, we determine the contributions of the intrinsic noise to the evolution equation for this simple pattern forming model.

\section{EROSION MODEL}

The model introduced in Ref. [9] considers an interface in $1+1$ dimensions described by the height variable $h_{i}(t)$, $i=1, \ldots, L$, where $L$ is the system size. The sites below the interface are occupied with particles, whereas the sites above are empty. The lattice unit distance along the horizontal direction is denoted by $a$. The derivation of the noisy KS equation can be generalized to $d+1$ dimensions but in the following we discuss the $1+1$ dimensional case.

The two basic physical mechanisms acting on the surface are erosion and diffusion. In the model, a particle at the interface is chosen randomly. Then, the particle is 
subjected to an erosion rule with probability $f$, and to a diffusion rule with probability $1-f$.

The erosion rule is as follows: the particle at $h_{i}$ is eroded (the corresponding lattice position emptied) with probability $P_{e} Y_{i}$ [17]. The quantity $P_{e}$ is computed as $1 / 7$ times the number of occupied sites in a box of size $3 \times 3$ centered at the chosen site, i.e., $P_{e}$ is the number of occupied nearest and next-nearest neighbors of the particle at $h_{i}$, and takes the values $1 / 7,2 / 7, \ldots, 1$. The definition of $P_{e}$ accounts for the unstable erosion mechanism which exists in the physical systems due to the finite penetration depth of the bombarding ions into the eroded substrate [18]. The efficiency of the sputtering process is measured by the sputtering yield [19]

$$
Y_{i}=Y\left(\varphi_{i}\right)=y_{0}+y_{1} \varphi_{i}^{2}+y_{2} \varphi_{i}^{4}
$$

with the local slope $\varphi_{i}=\tan ^{-1}\left(\left(h_{i+1}-h_{i-1}\right) / 2 a\right)$. In [9] $y_{0}, y_{1}$, and $y_{2}$ were chosen such that $Y_{i}(0)=0.5$, $Y_{i}(\pi / 2)=0$, and with a maximum value $Y_{i}\left(\varphi_{m}\right)=1$ for $\varphi_{m}=1 \mathrm{rad}$. In general one would merely require that $y_{0}>0, y_{1}>0$, and $y_{2}<0[19$.

The surface diffusion rule moves a particle at the interface from the top of column $k$ to the top of a randomly chosen nearest-neighbor column with the rates

$$
w_{k}^{ \pm}=\frac{1}{1+\exp \left(\beta \Delta \mathcal{H}_{k \rightarrow k \pm 1}\right)},
$$

where the energy is $\mathcal{H}=\left(J / b^{2}\right) \sum_{i=1}^{L}\left(h_{i}-h_{i+1}\right)^{2}$ [20], and $\Delta \mathcal{H}_{k \rightarrow k \pm 1}$ is the energy difference between the final and initial configurations; $J$ is a coupling constant, $b$ is the unit lattice spacing in the height direction, and $\beta$ is the inverse temperature.

\section{STOCHASTIC FORMALISM}

The interface configuration at a given time $t$ is specified by the set of column variables $H=\left\{h_{1}, h_{2}, \ldots, h_{L}\right\}$. The moments of the transition rates $W\left(H, H^{\prime}\right)$ in Eq. (1) are defined as follows:

$$
\begin{aligned}
K_{i}^{(1)} & =\sum_{H^{\prime}}\left(h_{i}{ }^{\prime}-h_{i}\right) W\left(H^{\prime}, H\right), \\
K_{i, j}^{(2)} & =\sum_{H^{\prime}}\left(h_{i}{ }^{\prime}-h_{i}\right)\left(h_{j}{ }^{\prime}-h_{j}\right) W\left(H^{\prime}, H\right), \\
K_{i_{1}, \ldots, i_{n}}^{(n)} & =\sum_{H^{\prime}}\left(\prod_{k=1}^{n}\left(h_{k}{ }^{\prime}-h_{k}\right)\right) W\left(H^{\prime}, H\right) .
\end{aligned}
$$

The first step in order to derive the Langevin equation is to transform the master equation (11) into a KramersMoyal partial differential equation (see, e.g., [21])

$$
\frac{\partial P}{\partial t}=\sum_{n=1}^{\infty} \frac{(-1)^{n}}{n !} \frac{\partial^{n}}{\partial h_{i_{1}} \ldots \partial h_{i_{n}}}\left(K_{i_{1}, \ldots, i_{n}}^{(n)} P\right)
$$

where a sum over repeated indices is assumed.

Next, one identifies a parameter $\Omega$ such that $\Omega \rightarrow \infty$ denotes the macroscopic limit. For $\left|h_{i}^{\prime}-h_{i}\right| \sim O(1 / \Omega)$, the ratio $K^{(n+1)} / K^{(n)}$ will typically be of order $O(1 / \Omega)$ 222. If the intrinsic fluctuations are sufficiently small then only the $K^{(1)}$ term in Eq. (6) will survive in the limit $\Omega \rightarrow \infty$ [23]. However, in our case the intrinsic fluctuations are relevant. Then, in the limit $\Omega \rightarrow \infty$ the Kramers-Moyal expansion reduces to the Fokker-Planck equation

$$
\frac{\partial P}{\partial t}=-\frac{\partial}{\partial h_{i}}\left(K_{i}^{(1)} P\right)+\frac{1}{2} \frac{\partial^{2}}{\partial h_{i} \partial h_{j}}\left(K_{i, j}^{(2)} P\right),
$$

which is obtained from Eq. (6) by keeping only the $K^{(1)}$ and $K^{(2)}$ terms 24].

The Fokker-Planck equation can be used in the further analysis of the system. However, only average quantities such as, e.g., $\left\langle h_{i}(t)\right\rangle=\sum_{H} h_{i} P(H, t)$, can be calculated. Therefore it is generally more convenient to recast Eq. (7) in an equivalent Langevin form for the height $h_{i}(t)$. In the Stratonovich interpretation, the Langevin equation associated to the Fokker-Planck equation is

$$
\frac{\partial h_{i}}{\partial t}=K_{i}^{(1)}+\eta_{i}
$$

where terms of order $O(1 / \Omega)$ have been neglected, cf. [23. In Eq. (8), $h_{i}$ is a continuous variable describing the dynamics of the fluctuations of the height configurations $\left\{h_{1}(t), \ldots, h_{L}(t)\right\}$, as obtained in the macroscopic limit $\Omega \rightarrow \infty$, cf. [22] (see also [5,20]). The term $\eta_{i}$ is a Gaussian white noise with average value equal to zero, and variance

$$
\left\langle\eta_{i}(t) \eta_{j}\left(t^{\prime}\right)\right\rangle=K_{i, j}^{(2)} \delta\left(t-t^{\prime}\right) .
$$

\section{DISCRETE LANGEVIN EQUATION}

We now apply the formalism described in the previous section to the erosion model in [9]. The transition rate for the erosion rule reads

$$
W_{e}\left(H, H^{\prime}\right)=\frac{f}{\tau} \sum_{k} P_{e} Y_{k} \delta\left(h_{k}^{\prime}, h_{k}-b\right) \prod_{j \neq k} \delta\left(h_{j}^{\prime}, h_{j}\right),
$$

where $\tau$ is the time scale. The erosion probability $P_{e}$ can be expressed in different forms. We choose

$$
P_{e}=\frac{1}{7}\left(5+\frac{a_{0}}{a^{2}} \nabla^{2} h_{i}+a_{1} \Theta\right),
$$

with the discrete Laplacian $\nabla^{2} h_{i}=h_{i+1}-2 h_{i}+h_{i-1}$, and constants $a_{0}$ and $a_{1}$. In Eq. (11) the Laplacian accounts for the physical mechanism of the box rule, and the $\Theta$ term accounts for the finite size of the box. Defining $\theta(x)=1$ for $x \geq 0$, and $\theta(x)=0$ for $x<0$, the $\Theta$ term can be written

$$
\begin{aligned}
\Theta= & -\theta\left(h_{i-1}-h_{i}-2 b\right)\left[h_{i-1}-h_{i}-b\right] \\
& -\theta\left(h_{i+1}-h_{i}-2 b\right)\left[h_{i+1}-h_{i}-b\right] \\
& +\theta\left(h_{i}-h_{i-1}-3 b\right)\left[h_{i}-h_{i-1}-2 b\right] \\
& +\theta\left(h_{i}-h_{i+1}-3 b\right)\left[h_{i}-h_{i+1}-2 b\right] .
\end{aligned}
$$


The $\Theta$ term in Eqs. (11) and (12) becomes effectively zero in the hydrodynamic limit of the model where the surface roughens in a way consistent with the KPZ universality class and the slopes are small along the interface. Also, from the numerical simulation [9] it is known that the qualitative behavior of the model does not change if the size of the box is enlarged [25]. The sputtering yield is obtained from Eq. (3) as

$$
Y_{i}=y_{0}+\frac{y_{1}}{a^{2}}\left(\nabla h_{i}\right)^{2}+\frac{y_{2}-2 y_{1} / 3}{a^{4}}\left(\nabla h_{i}\right)^{4}+\ldots,
$$

with the discrete gradient $\nabla h_{i}=\left(h_{i+1}-h_{i-1}\right) / 2$.

The transition rate for the diffusion rule reads

$W_{d}\left(H, H^{\prime}\right)=\frac{1-f}{2 \tau} \sum_{k}\left[w_{k}^{+} \delta\left(h_{k}^{\prime}, h_{k}-b\right) \delta\left(h_{k+1}^{\prime}, h_{k+1}+b\right)+w_{k+1}^{-} \delta\left(h_{k}^{\prime}, h_{k}+b\right) \delta\left(h_{k+1}^{\prime}, h_{k+1}-b\right)\right] \prod_{j \neq k, k+1} \delta\left(h_{j}^{\prime}, h_{j}\right)$,

where $w_{k}^{ \pm}$are defined in Eq. (四). By expanding the transition rates $w_{k}^{ \pm}$, we find

$$
w_{k}^{ \pm}=\frac{1}{1+q}-\frac{2 \beta J q / b}{(1+q)^{2}}\left(\nabla^{2} h_{k}-\nabla^{2} h_{k \pm 1}\right)+\sum_{n=2} \frac{C_{n}(q)}{b^{n}}\left(\nabla^{2} h_{k}-\nabla^{2} h_{k \pm 1}\right)^{n}
$$

where $q \equiv \exp (6 \beta J)$, and $C_{n}(q)$ are numerical constants whose exact value will not be needed in what follows.

Using that $W\left(H, H^{\prime}\right)=W_{e}\left(H, H^{\prime}\right)+W_{d}\left(H, H^{\prime}\right)$, and expression (5), we obtain to lowest order the following values for the transition moments:

$$
\begin{aligned}
& K_{i}^{(1)}=-\frac{f b}{\tau} P_{e} Y_{i}-\frac{(1-f) \beta J q}{\tau(1+q)^{2}} \nabla^{2}\left(\nabla^{2} h_{i}\right), \\
& K_{i, j}^{(2)}=-\frac{(1-f) b^{2}}{\tau(1+q)} \nabla^{2} \delta_{i j}+\frac{f b^{2}}{\tau} P_{e} Y_{i} \delta_{i j} .
\end{aligned}
$$

Here, $\nabla^{2} \delta_{i j}=\delta_{i+1, j}-2 \delta_{i, j}+\delta_{i-1, j}$. Higher order terms of the form $\left(\nabla^{2} h_{i}-\nabla^{2} h_{i \pm 1}\right)^{2 n+1}$ have been omitted (in section $\mathrm{V}$ we will argue that such terms are irrelevant in the renormalization group (RG) sense for the scaling properties of the interface). The quantity $P_{e} Y_{i}$ reads

$$
P_{e} Y_{i}=\frac{5 y_{0}}{7}+\frac{y_{0} a_{0}}{7 a^{2}} \nabla^{2} h_{i}+\frac{5 y_{1}}{7 a^{2}}\left(\nabla h_{i}\right)^{2},
$$

with additional terms of the form $\left(\nabla h_{i}\right)^{2 m} \nabla^{2} h_{i}$.

The different terms appearing in Eq. (16) give rise to the following contributions in the Langevin equation (8): an additive constant which corresponds to the average velocity of erosion for a flat interface, a negative Laplacian which reflects the unstable nature of the erosion rule, and a $\left(\nabla h_{i}\right)^{2}$ nonlinearity of the KPZ type describing lateral motion of the interface [15]; the nonlinearity originates from the coefficient $y_{1}$ in expression (3) for the sputtering yield. In addition, the diffusion rule contributes to Eq. (8) with the linear term $\nabla^{4} h_{i}$ (plus higher order nonlinearities). As demonstrated in Ref. [20] by numerical simulations and shown using a coarse-graining procedure, the $\nabla^{4} h_{i}$ term originates from the conserved nature of the surface diffusion rule (化) [5:20].

\section{CONTINUOUS LANGEVIN EQUATION}

To obtain a continuous Langevin equation, we assume that the discrete function $h_{i}(t)$ can be replaced by a smooth function $h(x, t)$ such that $h_{i}(t)=h(x=i a, t)$, and further that

$$
h_{i \pm 1}(t)-h_{i}(t)=\left.\sum_{n=1}^{\infty} \frac{( \pm a)^{n}}{n !} \frac{\partial^{n} h}{\partial x^{n}}\right|_{x=i a} .
$$

A more rigorous way to arrive at the function $h(x, t)$ would be through some coarse graining procedure which preserves the symmetry of the problem. However, as discussed in [5.20], this is a highly nontrivial task. Here we are concerned with the form of the relevant terms in the equation of motion for $h(x, t)$, which are expected to have the same form as those obtained by the use of (18). We will determine them as the leading terms in an expansion in which we take the parameters $a$ and $b$ to be small but nonzero [27]. Combining (18) with Eqs. (8), (9) we obtain the continuous Langevin equation for $h(x, t)$ :

$$
\begin{aligned}
\frac{\partial h}{\partial t}= & v_{0}-|\nu| \nabla^{2} h-\kappa\left(\nabla^{2}\right)^{2} h+\frac{\lambda}{2}(\nabla h)^{2}+\eta(x, t) \\
& +c_{1}(\nabla h)^{2} \nabla^{2} h+c_{2}\left(\nabla^{3} h\right)^{2} \nabla^{4} h+\ldots
\end{aligned}
$$

where now $\nabla \equiv \partial / \partial x$. The noise $\eta(x, t)$ has correlations

$$
\begin{aligned}
\left\langle\eta(x, t) \eta\left(x^{\prime}, t^{\prime}\right)\right\rangle & =\left[2 D-2 D_{d} \nabla^{2}\right] \delta\left(x-x^{\prime}\right) \delta\left(t-t^{\prime}\right) \\
& +\left[D_{1} \nabla^{2} h+D_{2}(\nabla h)^{2}\right] \delta\left(x-x^{\prime}\right) \delta\left(t-t^{\prime}\right) .
\end{aligned}
$$

The coefficients in Eqs. (19)-200 read

$$
\begin{array}{rlrl}
v_{0} & =-\frac{5 f y_{0} b}{7 \tau}, & |\nu|=\frac{f y_{0} a_{0} b}{7 \tau}, \quad \kappa=\frac{(1-f) \beta J q a^{4}}{\tau(1+q)^{2}}, \\
\lambda & =-\frac{10 f y_{1} b}{7 \tau}, \quad D=\frac{5 f y_{0} a b^{2}}{14 \tau}, & D_{d}=\frac{(1-f) a^{3} b^{2}}{2 \tau(1+q)^{2}}, \\
D_{1}=\frac{f y_{0} a a_{0} b^{2}}{7 \tau}, & D_{2}=\frac{5 f y_{1} a b^{2}}{7 \tau} .
\end{array}
$$

The terms in Eq. (19a) constitute the noisy KuramotoSivashinsky equation (2), where one notes the presence of a negative Laplacian and a KPZ nonlinearity. The presence of such a nonlinearity is known to determine the 
scaling behavior of the noisy KS equation as shown by an RG analysis [26]. Using the values of the roughness and dynamic exponents at the KPZ fixed point 15], the terms appearing in Eq. (19b) can be shown to be irrelevant. A similar RG argument shows the irrelevance of the conserved term $D_{d}$ in the noise correlator (20) in the presence of the shot noise $D$, as well as the irrelevance of the multiplicative contributions $D_{1}$ and $D_{2}$.

\section{CONCLUSIONS}

In summary, we have derived the continuum equation for a microscopic model for ion sputtering. The resulting equation is a noisy version of the Kuramoto-Sivashinsky equation. Such an equation exhibits the same scaling

[1] J. Krug and H. Spohn, "Kinetic Roughening of Growing Surfaces", in Solids far from Equilibrium: Growth, Morphology and Defects, ed. C. Godrèche (Cambridge University Press, Cambridge, 1991).

[2] A.-L. Barabási and H. E. Stanley, Fractal Concepts in Surface Growth (Cambridge University Press, Cambridge, 1995).

[3] T. Halpin-Healey and Y.-C. Zhang, Phys. Rep. 254, 215 (1995).

[4] M. Plischke, Z. Rácz, D. Liu, Phys. Rev. B 35, 3485 (1987).

[5] Z. Rácz, M. Siegert, D. Liu, and M. Plischke, Phys. Rev. A 43, 5275 (1991).

[6] D. D. Vvedensky, A. Zangwill, C. N. Luse, and M. R. Wilby, Phys. Rev. E 48, 852 (1993).

[7] K. Park and B. Kahng, Phys. Rev. E 51, 796 (1995).

[8] See Refs. [ [ [5] wherein difficulties with respect to the continuum limit are discussed.

[9] R. Cuerno, H. A. Makse, S. Tomassone, S. T. Harrington, and H. E. Stanley, Phys. Rev. Lett. 75, 4464 (1995).

[10] Y. Kuramoto and T. Tsuzuki, Prog. Theor. Phys. 55, 356 (1977); G. I. Sivashinsky, Acta Astronaut. 6, 569 (1979).

[11] M. C. Cross and P. C. Hohenberg, Rev. Mod. Phys. 65, 851 (1993).

[12] S. Zaleski, Physica D 34, 427 (1989).

[13] K. Sneppen, J. Krug, M. H. Jensen, C. Jayaprakash, and T. Bohr, Phys. Rev. A 46, R7351 (1992).

[14] V. L'vov, V. V. Lebedev, M. Paton, and I. Procaccia, Nonlinearity 6, 25 (1993).

[15] M. Kardar, G. Parisi, and Y.-C. Zhang, Phys. Rev. Lett. 56, 889 (1986). properties as the Kardar-Parisi-Zhang equation and this result is in nice agreement with the simulations of the erosion model performed in Ref. [9]. The master equation approach has allowed us to derive the form of the stochastic noise in the Langevin equation for a simple pattern forming system.

\section{ACKNOWLEDGEMENTS}

We acknowledge discussions with S. Tomassone and H. E. Stanley. K. B. L. acknowledges the support from the Danish Natural Science Research Council, and R. C. acknowledges support from Ministerio de Educación y Ciencia, Spain. The Center for Polymer Studies is supported by NSF.

[16] A. Karma and C. Misbah, Phys. Rev. Lett. 71, 3810 (1993).

[17] In Ref. 9] the erosion rule was implemented in a different way which, however, is equivalent to the form given here.

[18] P. Sigmund, J. Mat. Sci. 8, 1545 (1973); R. M. Bradley and J. M. E. Harper, J. Vac. Sci. Technol. A 6, 2390 (1988); R. Cuerno and A.-L. Barabási, Phys. Rev. Lett. 74, 4746 (1995).

[19] G. Carter, B. Navinšek, and J. L. Whitton, in Sputtering by Particle Bombardment, R. Behrisch, ed., Vol. II (Springer-Verlag, Heidelberg, 1983).

[20] M. Siegert and M. Plischke, Phys. Rev. E 50, 917 (1994).

[21] H. Risken, The Fokker-Planck Equation: Methods of Solution and Applications, 2nd ed. (Springer-Verlag, Heidelberg, 1989).

[22] R. F. Fox, Phys. Rep. 48, 179 (1978).

[23] R. F. Fox and J. Keizer, Phys. Rev. A 43, 1709 (1991).

[24] The Fokker-Planck equation (耳) will be correct to order $O(\ln \Omega / \Omega)$, cf. 23.

[25] In such a case, say for a $3 \times N$ box, the change in $P_{e}$ in Eq. (11) affects merely the constant term and the $\Theta$ term which becomes effectively zero for even earlier stages of the dynamics than in the $3 \times 3$ case.

[26] R. Cuerno and K. B. Lauritsen, Phys. Rev. E 52, 4853 (1995).

[27] In the discrete model, $b$ is the analog of the finite penetration depth of the arriving ions into the solid substrate, while $a$ would correspond to the width of the spatial distribution of energy deposited by a bombarding ion within distance $b$ from the surface. Both quantities represent a lower wavelength cutoff or spatial resolution whose typical values for physical systems are of the order of $100 \AA$ [18]. 